Original research article

\title{
JNK inhibitor CC-930 reduces fibrosis in a murine model of $N f 1$-deficient fracture repair
}

\author{
Nikita Deo ${ }^{\mathrm{a}, \mathrm{b}}$, Jad El-Hoss $^{\mathrm{a}, \mathrm{b}}$, Mille Kolind ${ }^{\mathrm{a}}$, Kathy Mikulec ${ }^{\mathrm{a}}$, Lauren Peacock ${ }^{\mathrm{a}}$, \\ David G. Little ${ }^{\mathrm{a}, \mathrm{b}}$, Aaron Schindeler ${ }^{\mathrm{a}, \mathrm{b}, *}$ \\ a The Children's Hospital at Westmead, Orthopaedic Research \& Biotechnology Unit, Sydney, Australia \\ ${ }^{\mathrm{b}}$ University of Sydney, Sydney Medical School, Discipline of Paediatrics and Child Health, Sydney, Australia
}

\section{A R T I C L E I N F O}

\section{Article history:}

Received 16 March 2017

Received in revised form 3 January 2018

Accepted 17 January 2018

Available online 13 February 2018

\section{Keywords:}

NF1

Neurofibromatosis

Pseudarthrosis

BMP-2

JNK

CC-930

\begin{abstract}
A B S T R A C T
Tibial pseudarthrosis often features deficient bone formation, excessive bone resorption, and extensive pathological fibrosis, particularly in individuals with Neurofibromatosis type I (NF1). It was hypothesized that overactive NF1-Ras-JNK signalling may underlie the pathological fibrosis, and that this could be treated via a JNK antagonist. CC-930, a small molecule JNK inhibitor, was trialed in closed fractures in wild type mice CC-930 (25 mg/kg/twice daily) was dosed throughout fracture healing (D2-21) and during the latter stages of repair (D11-21). All fractures healed by D21, regardless of treatment, with some of the CC930 (D11-21) treatment group showing early bridging. CC-930 (D11-21) was tested in an Nf1-null fracture model where $N f 1$ was inactivated by Ad-Cre virus injection in $N f 1^{\text {flox/flox }}$ mice; these mice also possessed a Cre-responsive tdTomato transgene. CC-930 resulted in a significant decrease in non-unions (93\% vehicle $v s .64 \%$ CC-930, $p<0.01)$. Local treatment with the bone anabolic rhBMP-2 $(10 \mu \mathrm{g})$ increased union and callus bone volume, but also increased the fibrotic tissue at the fracture site. Fractures treated with a combination of rhBMP-2 $(10 \mu \mathrm{g})$ and CC-930 were all partially or fully bridged by D21 ( $p<0.01 v s$. vehicle) and there was a significant decrease in fibrosis vs. rhBMP-2 alone $(p<0.01)$. In untreated $N f 1$ null fractures, the tdTomato transgene was expressed in fibrous tissue at the fracture site, but not in the newly forming bone. These data suggest that JNK inhibition may be an effective therapeutic approach for reducing pathological fibrosis in NF1 tibial pseudarthrosis, however other adjunctive strategies may be required to augment bone formation.

Crown Copyright (c) 2018 Published by Elsevier Sp. z o.o. on behalf of Faculty of Health and Social Sciences,
\end{abstract} University of South Bohemia in Ceske Budejovice. All rights reserved.

\section{Introduction}

Neurofibromatosis type 1 (NF1) is an autosomal dominant genetic disease that has been estimated to affect approximately 1 in 2500 individuals (Evans et al., 2010). Upwards of 50\% of children with NF1 exhibit skeletal abnormalities ranging from reduced bone mineral density (BMD) and sub-clinical bone dysplasias to tibial dysplasia and scoliosis (Schindeler and Little, 2008; Stevenson et al., 2007). Although an uncommon complication of NF1, tibial dysplasia is particularly challenging to manage and while bracing can delay fracture, in most cases the dysplastic bone eventually goes on to break. The subsequent impaired bone healing

\footnotetext{
* Author for correspondence: The Children's Hospital at Westmead, Research Building, Orthopaedic Research \& Biotechnology, Locked Bag 4001, Westmead, NSW 2145, Australia.

E-mail address: aaron.schindeler@sydney.edu.au (A. Schindeler).
}

typically results in a persistent non-union called a pseudarthrosis (Hefti et al., 2000). Surgical intervention to correct a pseudarthrosis involves removal of infiltrating fibrotic tissue, correction of angular deformities and mechanical stabilization, and promotion of repair via autologous bone grafting and/or recombinant human bone morphogenetic proteins (rhBMPs) (Stevenson et al., 2013). Even with surgery, NF1 pseudarthroses often have a poor prognosis and commonly result in amputation or residual disability. There have been many recent advancements in understanding the molecular and genetic basis of this pathophysiology. Genetic analyses of biopsied pseudarthrotic tissue taken during surgery indicate that localized double inactivation of the NF1 gene is an important trigger for dysplasia and fibrosis (El-Hoss et al., 2012; El Khassawna et al., 2012; Stevenson et al., 2006).

The NF1 gene encodes the protein neurofibromin, a negative regulator of the small GTPase, Ras. Both the skeletal and nonskeletal manifestations of NF1 have been attributed to the resultant upregulation of Ras signalling (Elefteriou et al., 2006; 
Kolanczyk et al., 2008). Multiple kinase pathways are downstream of Ras, and the relative significance of these pathways is likely to vary between cell and tissue types. The Ras-MEK-ERK1/2 axis has been noted as particularly important not only tumour growth but also bone anabolism. But while MEK inhibition with PD0325901 increased callus bone volume in an $N f 1^{\text {null }}$ murine tibial fracture model, it did not prevent the accrual of excess fibrous tissue (El-Hoss et al., 2014b; Sharma et al., 2013).

The c-Jun $\mathrm{NH}_{2}$-terminal kinase (JNK) pathway is a stressactivated protein signalling cascade belonging to the Ras-MAPK superfamily (Johnson and Lapadat, 2002). JNKs are important regulators of normal physiological immune responses and tissue morphogenesis (Davis, 2000; Tournier et al., 2000). The physiological relevance of JNK inhibition has previously been demonstrated in animal models of inflammatory rheumatoid arthritis (Han et al., 2001) and idiopathic pulmonary fibrosis (Rafii et al., 2013). In the context of NF1, JNK activity has been reported to be up-regulated in $\mathrm{Nf}^{+/-}$microglial cells, and treatment with a JNK inhibitor was shown to rescue elevated cell proliferation and proinflammatory cytokine production (Daginakatte et al., 2008). In cultured Nf1-deficient and Nf1-null osteoblasts, JNK signalling was similarly over-activated, and treatment with a JNK inhibitor increased the expression of osteogenic markers in these cells (Sullivan et al., 2011). Based on these data it was it hypothesized that JNK inhibitor treatment may improve NF1 deficient bone repair both by inhibition of fibrosis and promotion of osteogenic differentiation.

Prior studies using MEK inhibitors in fracture repair models have demonstrated the importance of the timing of inhibitor dosing on fracture repair outcome. For example, during normal fracture healing, MEK inhibitor impairs the removal of the cartilaginous soft callus, however this agent has no adverse effects on the initial phases of inflammation and soft callus formation (El-Hoss et al., 2014b). For this reason, MEK inhibitor treatment in the $N f 1^{\text {null }}$ tibial fracture model was restricted to the initial phases of fracture healing (El-Hoss et al., 2014b). Thus investigating the ideal timing for JNK inhibition was identified as important for obtaining optimal results.

In this study we examined the effects of CC-930, a small molecule inhibitor of JNK signalling (Plantevin Krenitsky et al., 2012). First we employed a simple model of tibial closed fracture repair in wild type mice; this model heals normally without intervention in 2-3 weeks (Schindeler et al., 2008a). In this system we trialed continuous dosing (D2-21) versus treatment restricted to late fracture healing (D11-21). Next, we utilized an established model of NF1 deficient bone repair that uses a Cre-recombinase expressing adenovirus (Ad-Cre) in an $\mathrm{Nf}^{\text {flox/flox }}$ mouse to produce local double inactivation of the Nf1 locus (El-Hoss et al., 2012). This model features substantive fibrosis and poor repair. The primary outcome measure for both of these studies was fracture union, and the effects on fibrous tissue and callus bone volume were key secondary outcomes.

\section{Materials and methods}

\section{Animals}

10-12 week old $N f 1^{\text {flox/flox }}$ mice and 10 -week wild type C57BL/6 were used in fracture studies. $N f 1^{\text {flox/flox }}$ mice were sourced from the National Cancer Institute (NCI) mouse repository (Bethesda, Washington DC, USA) from the strain originally generated by Prof $\mathrm{L}$. Parada (Zhu et al., 2001). Wild type C57BL/6 mice were purchased from the Animal Resources Center (Perth, Australia). Mice containing the Ai9 tdTomato fluorescent reporter (B6.Cg-Gt (ROSA)26Sor $\left.{ }^{\text {tm9(CAG-tdTomato }) H z e / J}\right)$ were obtained from the Jackson
Laboratory mouse repository (Sacramento, CA, USA) (Madisen et al., 2010).

Mice were housed in the institutional transgenic facility in sterile polypropylene boxes and stainless steel cage lids with food and water supplied ad libitum. Animals receiving oral drugs in edible jelly were singly housed for the duration of the dosing to ensure the correct dose was received. All animal experiments were approved by the CHW/CMRI institutional Animal Ethics Committee under protocol K319. This study was consistent with the ARRIVE guidelines.

\section{Adenovirus production}

Adenovirus for in vivo experiments was produced in-house using cultured HEK cells infected with a stock virus (Zha et al., 2008) after 72-96 h using previously described methods (El-Hoss et al., 2012). The ultracentrifuge purified and dialyzed virus was titered using an Adeno- $\mathrm{X}^{\mathrm{TM}}$ Rapid Titer kit (Clontech, Mountain View, CA, USA) and stored at $-80^{\circ} \mathrm{C}$ in $10 \%$ glycerol.

\section{Fracture model and surgery}

Fracture surgery was performed by a single experienced operator with an assistant for anesthesia and post-operative suturing. Mice received tibial mid-shaft fractures as detailed in the original $N f 1$ fracture knockout paper (El-Hoss et al., 2012). In brief, anesthesia was induced using a combination of $35 \mathrm{mg} / \mathrm{kg}$ ketamine and $4.5 \mathrm{mg} / \mathrm{kg}$ xylazine delivered by intra-peritoneal injection and maintained with inhaled isoflurane gas. Unilateral fractures were produced manually by 3 -point bending using a handheld surgical staple remover then internally stabilized using a sterile $0.3 \mathrm{~mm}$ diameter stainless steel insect pin. Post-operative X-rays were taken to confirm fracture position and stability using a digital X-ray machine (Faxitron Bioptics LLC, Tucson, ZA, USA).

In $N f f^{\text {flox/flox }}$ mice, local double inactivation of the $N f 1$ locus was achieved via local delivery of Ad-cre virus using a published model (El-Hoss et al., 2012; Schindeler et al., 2008b). Local dosing of $1 \times 10^{8}$ infectious units of purified Ad-Cre virus was delivered by absorbable collagen sponge (ACS, Medtronic Australasia, North Ryde, NSW, Australia) in an elaboration of the previously published technique. The collagen sponge was wrapped around the fracture site. Animals treated with rhBMP-2 received $10 \mu \mathrm{g}$ co-delivered in the collagen sponge (rhBMP-2/ACS, Medtronic Australasia) (El-Hoss et al., 2014a). In Ai9 (tdTomato fluorescent reporter mice) Ad-Cre virus injected into closed fractures as previously described (El-Hoss et al., 2012). Animals were monitored and underwent weekly $\mathrm{x}$-rays post-surgery to longitudinally assess fracture healing and union.

\section{JNK inhibitor dosing}

Prior experiments using MEK inhibitors demonstrated that the effects of pathway inhibition on fracture repair could be highly dependent on the timing of inhibitor dosing (El-Hoss et al., 2014b; Madisen et al., 2010). Initial experiments in wild type mice were performed to examine the effects of JNK inhibitor CC-930 on fracture repair given at different times. CC-930 (Celgene Corporation, Summit, NJ, USA) was administered twice daily at a dose rate of $25 \mathrm{mg} / \mathrm{kg}$ solubilized and added to flavoured jelly: 16\% Splenda ${ }^{\mathbb{R}}$ (Splenda ${ }^{\mathbb{R}}$ Low Calorie Sweetener, Johnson-Johnson Pacific Pty, NSW, Australia), 9.6\% gelatine (Davis Gelatin, GELITA Australia Pty, NSW, Australia) and 7.9\% flavouring (QUEEN Flavouring Essence Imitation Strawberry, Queen Fine Foods Pty, QLD, Australia) (El-Hoss et al., 2014b). With day 0 defined as the day of fracture surgery, CC-930 was administered from D2-21 (Continuous treatment) or D11-21 (Late treatment). 
Micro-CT analysis of bone volume

Samples were scanned in 70\% ethanol using a SkyScan 1174 compact micro-computed tomography (micro-CT) scanner (Kontich, Belgium). Specimens were scanned using a $0.5 \mathrm{~mm}$ aluminium filter, $50 \mathrm{kV}$ X-ray tube voltage, and $800 \mu \mathrm{A}$ tube electric current. Scanned images were reconstructed using NRecon, version 1.6.1.7 (SkyScan), and analyzed using CTAnalyser software, version 1.11.8.0 (SkyScan).

Bitmaps of scanned images were created and then analyzed by using a ROI manually selected to include the fracture callus and exclude the old bone cortex. Callus bone volume (BV) and callus tissue volume (TV) were quantified and used to calculate overall $\mathrm{BV} / \mathrm{TV}$ ratios.

\section{Histological analysis}

Fractured tibiae were fixed in 4\% PFA/PBS solution overnight then transferred to $70 \%$ ethanol. Prior to embedding, fractures were transferred to $30 \%$ sucrose/PBS overnight at $4{ }^{\circ} \mathrm{C}$ to ensure optimal cryopreservation. Fractures were embedded in TissueTek ${ }^{\circledR}$ Optimal Cutting Temperature media (Sakura Finetek, The Netherlands) and $5 \mu \mathrm{m}$ thick longitudinal frozen sections of undecalcified bone tissue were obtained using a CM1900 cryostat (Leica Microsystems, North Ryde, Australia) on non-fluorescent cryo-tape. Sections were stained with Hematoxylin/Eosin, Picrosirius Red/Alcian Blue or Tartrate Resistant Acid Phosphatase (TRAP) stain, as carried out on $5 \mu \mathrm{m}$ thick sections of paraffin embedded tibiae as previously described (Schindeler et al., 2008b). Multiple slides were mounted and then quantified using the
Bioquant Image Analysis system (Bioquant Image Analysis Corp, Nashville, TN, USA).

Frozen, fixed and undecalcified histological $5 \mu \mathrm{m}$ sections were obtained from the Ai9 tdTomato reporter mice at the completion of the D21 healing time-point to visualize virus recombination at the fracture site. Samples were embedded and sectioned as previously described, and directly mounted in a $50 \%$ glycerol/PBS solution. The fluorescent signal was visualized using an EX50 correlative light microscope (Olympus).

\section{Cell culture and western blotting}

Primary fibroblasts were obtained from 1 to 2 week old Ai9 $\times N f 1^{\text {flox/flox }}$ neonates. Cells were grown in high serum DMEM (20\% fetal bovine serum) with antibiotics (1\% penicillin/streptomycin) and $1 \%$ glutamine. Once confluent, cells were passaged for experimental usage. Semi-confluent cells were serum-starved overnight and then treated with purified adenovirus-Cre in Gene Jammer transfection reagent to produce $\mathrm{Nf1^{-l- }}$ fibroblasts. Cells were differentiated in osteogenic (OS) media consisting of $\alpha$-MEM, $10 \%$ fetal bovine serum with $50 \mu \mathrm{g} / \mathrm{ml}$ ascorbic acid and $1 \mathrm{mM}$ $\beta$-glycerophosphate. Alternatively, explanted fractures were grown in OS media with or without $5 \mu$ M CC-930.

Cells were stained using Alizarin Red S as previously described (Schindeler et al., 2010). For JNK activity analysis, cells from explanted calluses had proteins extracted via the RIPA buffer method with phosphatase and protease inhibitors (Sigma Aldrich) and resolved in 10\% Tris/Glycine gels (Bio-Rad Laboratories, Inc.) by SDS/PAGE gel electrophoresis. Protein membranes were incubated with phospho-c-Jun (Ser73) (Cell Signalling Technology, Inc.) and

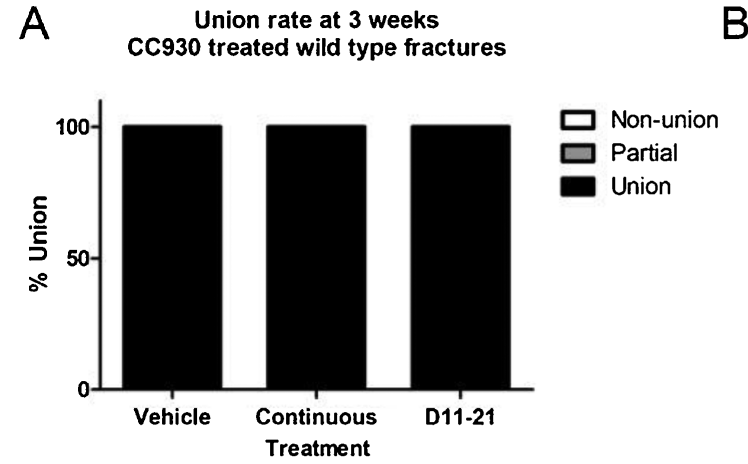

C Bone Volume in Cc930 treated
wild type fractures

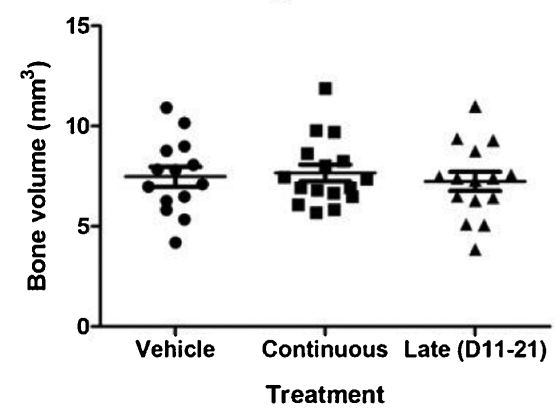

B

D

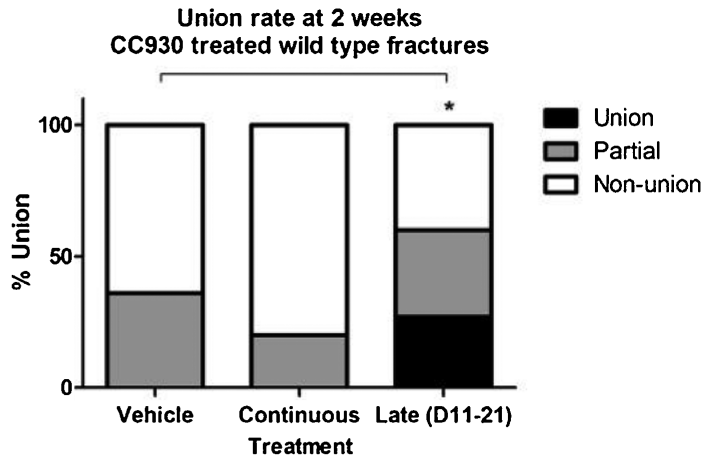

\section{BV/TV in CC930 treated wild type fractures}

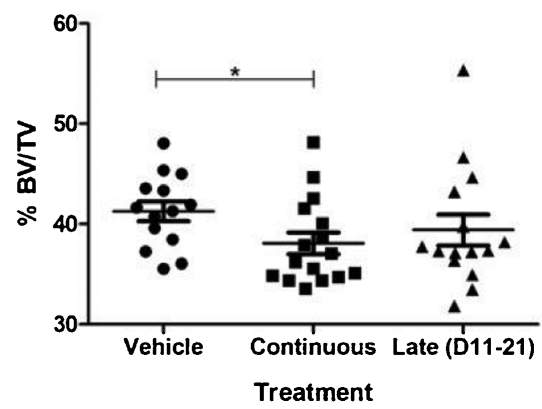

Fig. 1. Radiographic analysis of wild type, closed tibial fractures treated with CC-930. (A) In all wild type mice, fractures (treated and untreated) were united by 3 weeks. (B) At 2 weeks, there was a significant increase in radiographic union in the mice treated with late CC-930 (D11-21) ( $\left.{ }^{*} P=0.04\right)$. (C) Analysis of the bony callus by microCT showed no significant difference in BV with CC-930 treatment compared to vehicle control in wild type mice. (D) Callus BV/TV was significantly decreased ( $P<0.01$ ) with continuous CC-930 treatment. 
c-Jun (Ser73) (Cell Signalling Technology, Inc.) with a dilution of $1: 1000$.

\section{Statistical analysis}

Statistical analyses and graphs were generated using GraphPad Prism software (La Jolla, USA). For fracture union, a Fisher's exact test was used to analyze union rate between treatment groups ( $n=15$ /group) and an untreated control group ( $n=15$ ) The number of mice used per treatment group was calculated to have sufficient statistical power to detect a 50\% reduction in non-union in the Nf1-null fracture model. One-way ANOVA parametric statistical tests were performed to analyze micro-CT values and a two-tailed student's $t$-test was used to analyze tissue histology values. A cut-off of $p<0.05$ was considered statistically significant.

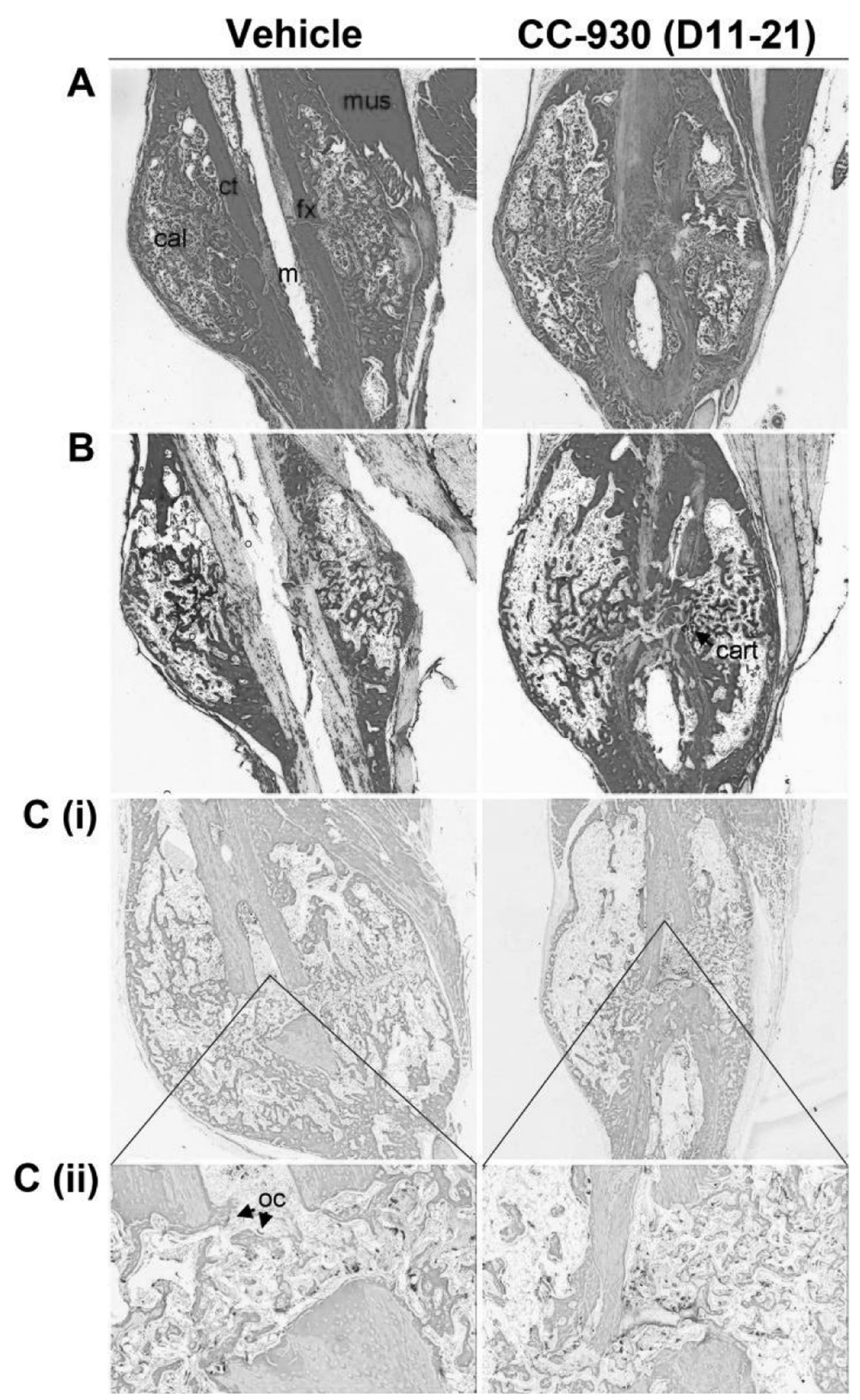

Fig. 2. Histological analysis of wild type closed tibial fractures treated with CC-930. Histological specimens representing the median callus BV for each group were stained with (A) Hematoxylin/Eosin, (B) Picrosirius red/Alcian blue, (C) Tartrate-resistant acid phosphatase (TRAP). No discernible differences were seen in callus architecture, and no meaningful retention of soft cartilaginous callus was seen in the CC-930 treated specimens. TRAP+ cells appeared modestly increased in the CC-930 specimens, but this was not quantifiable. Labels: [cal] bony callus, [ct] old tibial cortex, [fx] fracture site, [m] medullary canal, [mus] muscle, [cart] cartilage remnant, [oc] TRAP+ osteoclast on the bone surface. 


\section{Results}

\section{CC-930 treatment does not impair closed tibial fracture healing}

The effect of the CC-930 JNK inhibitor was first investigated in the context of normal fracture healing. Wild type C57BL/6 mice underwent closed tibial fractures, which normally unite between 2 and 3 weeks post-fracture. Dosing regimens all utilized $25 \mathrm{mg} / \mathrm{kg}$ twice daily CC-930 delivered orally via an edible jelly $(n=15$ per group). Comparison of this delivery system with standard gavaging protocols demonstrated similar rapid clearance of the drug, but superior initial bioavailability (Supp. Table 1).

Radiographic assessment (X-ray) of all fractures confirmed that all fractures were healed by D21 post-surgery (Fig. 1A), indicating that CC-930 did not substantively impair bone healing under normal physiological conditions. Analysis of the effects of CC-930 on the early stages of fracture healing by examining D14 union rates indicated that dosing from D2-14 (continuous group) did not significantly improve or impair bone healing. In contrast, improved union rate was seen in the late treatment group, which had received CC-930 from D11-D14 (Fig. 1B).

Consistent with radiographic healing in all mice, microCT analysis of the fracture callus showed robust bone formation. BV (Fig. 1C) and BV/TV (Fig. 1D) were comparable between all treatment groups at D21. The continuous CC-930 group showed a minor but statistically significant reduction in BV/TV, but with mean BV/TV values all lying between 35 and 40\% this was not deemed to be biologically significant.

Tissue histology showed robust bone formation with Hematoxylin/Eosin and Picrosirius red/Alcian blue staining (Fig. 2A-B). By D21 the fractures were completely bridged and the hard callus in the process of remodelling to form a new cortex. At this time the old cortical bone was still present. No evidence of residual cartilage or fibrosis was seen in wild type fractures at D21. Examination of osteoclasts using TRAP staining showed a higher number of osteoclasts in the fracture gap of some CC-930 samples (Fig. 2C), however these data were variable and not consistently higher with JNK inhibitor treatment.

\section{CC-930 and rhBMP-2 enhance Nf1null tibial fracture healing}

Interventions were trialed in the $N f 1^{\text {null }}$ tibial fracture model that features delayed union and extensive fibrosis. In addition to CC-930 (D11-21) treatment, some groups received local delivery of $10 \mu \mathrm{g}$ rhBMP-2 during surgery to enhance bone formation. The largest callus sizes were seen in groups receiving rhBMP-2 (Fig. 3A). The primary outcome measure of the study was radiographic union measured at D21 (Fig. 3B).

CC-930 led to a significant decrease in the non-union rate to $37 \%$, compared to $67 \%$ in vehicle treated controls $(P<0.01)$. Treatment with rhBMP-2, previously established to be effective in this model, gave a non-union rate of $14 \%$, and the combination of rhBMP-2 and CC-930 led to zero (0\%) non-unions (both $P<0.01 \mathrm{vs.}$ controls). The combination of rhBMP-2 and CC-930 was superior to CC-930 alone $(P<0.05)$. In control mouse fibroblasts in culture, the combination of rhBMP-2 and CC-930 similarly led to a promotion of matrix mineralization (Supp. Fig. 1A).

Supp. Fig. 1. (A) Embryonic fibroblasts from C57BL/6 mice were cultured in osteogenic (OS) media until semi-confluent then treated with CC-930 (5 $\mu \mathrm{M})$, rhBMP-2 (200 ng/ml) or both agents in combination. Cell monolayers were stained with Alizarin Red $\mathrm{S}$ for mineralization after 7 days. (B) Explanted tissue from fracture calluses from $n=3 \mathrm{Nf1}$-null (Ad-Cre treated) fractures were cultured in OS media $\pm 5 \mu \mathrm{M}$ CC-930. Analysis by western blot showed a significant inhibition of p-c-jun (p-JNK) relative to total
A

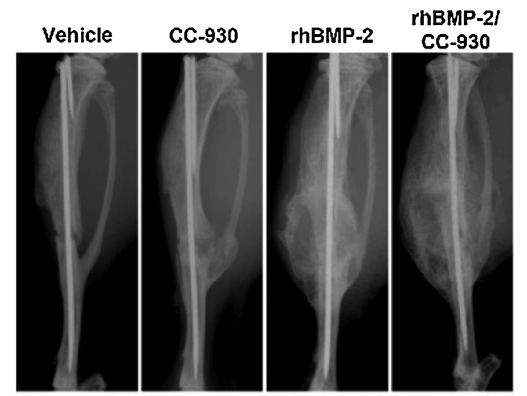

B

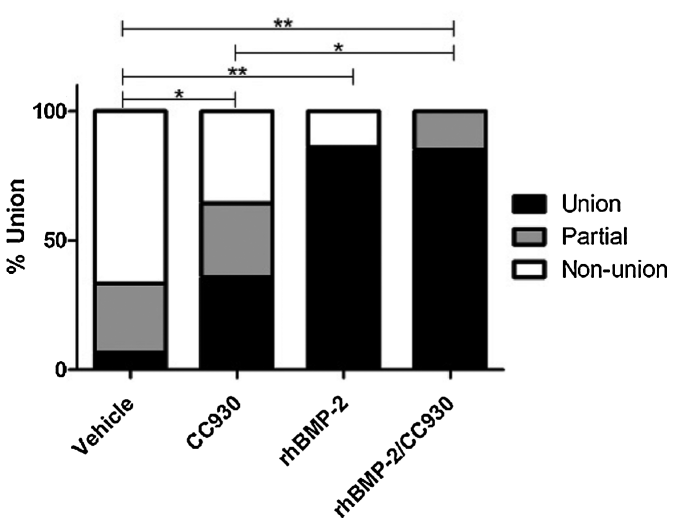

Fig. 3. CC930 and rhBMP-2 enhance fracture repair in a model of $N f 1$-null fracture healing. (A) Representative $x$-ray images of 3 week healing in each treatment group showing greatly enhanced bone formation with rhBMP-2 treatment; (B) Radiographic union rates after 3 weeks of healing with CC-930 were compared to vehicle treated controls. Significant increases in union were seen with all treatments versus controls, with the rhBMP-2 and CC-930 (D11-21) co-treatment producing the optimal results $\left({ }^{*} \mathrm{P}<0.05,{ }^{* *} \mathrm{P}<0.01\right)$.

c-jun (total JNK) in 2 of 3 CC-930 treated samples by Western blot. The robust band in the remaining lane could not be accounted for.

To examine callus bone volume and microarchitecture, microCT analysis was performed. MicroCT reconstructions illustrated the magnitude of the increased callus size with rhBMP-2 treatment (Fig. 4A) compared to controls. Quantification of callus BV reflected a 4-5 fold increase in bone with rhBMP-2 treatments compared to no rhBMP-2 groups (Fig. 4B). CC-930 treatment did not significantly affect callus BV. However, rhBMP-2 induced bone showed reduced BV/TV compared to the no rhBMP-2 groups, and again CC-930 did not impact BV/TV (Fig. 4C).

\section{CC-930 reduces fibrosis in Nf1null tibial fractures}

While CC-930 did not significantly impact on new bone formation, the impact on fibrosis in the $N f 1^{\text {null }}$ fracture model was examined via tissue histology. At the D21 time point, residual fibrous tissue and cartilaginous tissue was analyzed in tissue sections. While the majority of non-osseous tissue had undergone endochondral ossification by this time point, small amounts of both tissues remained.

Examination of fibrotic tissue indicated that rhBMP-2 increased fibrosis and CC-930 decreased fibrosis. It was found that the amounts of fibrotic and cartilaginous tissue could vary greatly between samples. The CC-930 group showed a $\sim 5$-fold reduction in fibrotic tissue compared to the rhBMP-2 group $(P=0.02)$, highlighting the different outcomes between pro-fibrotic and antifibrotic interventions (Fig. 5A). Moreover, the increases in fibrosis seen with rhBMP-2 could be significantly reduced by co-treatment with CC-930 $(P<0.01)$.

Analysis of cartilaginous tissue showed that the total remaining cartilage in $N f 1^{\text {null }}$ fractures was extremely low overall at D21 $(\sim 2 \%$ 
A

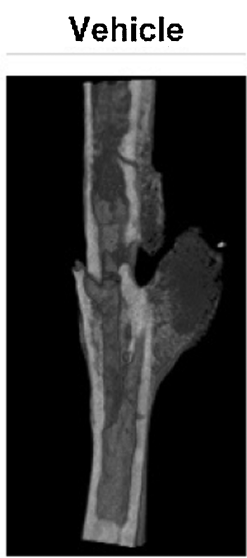

B

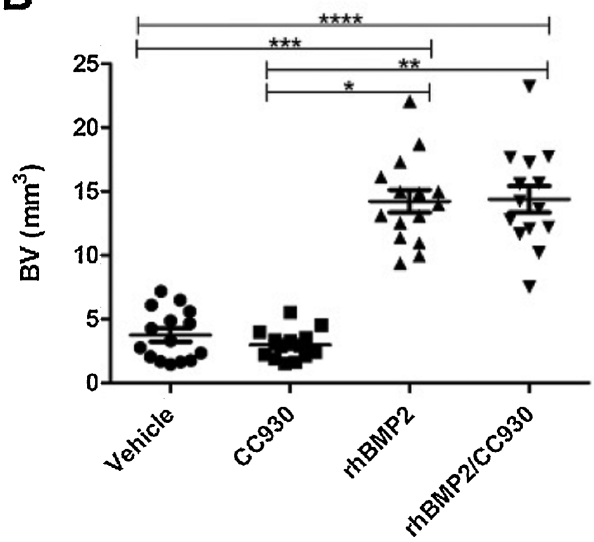

CC -930

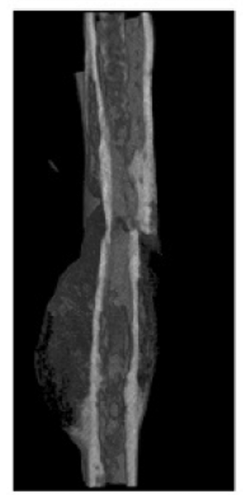

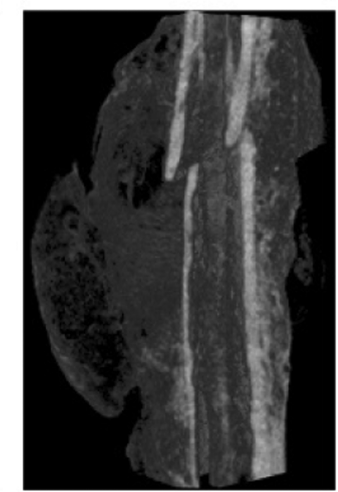

C

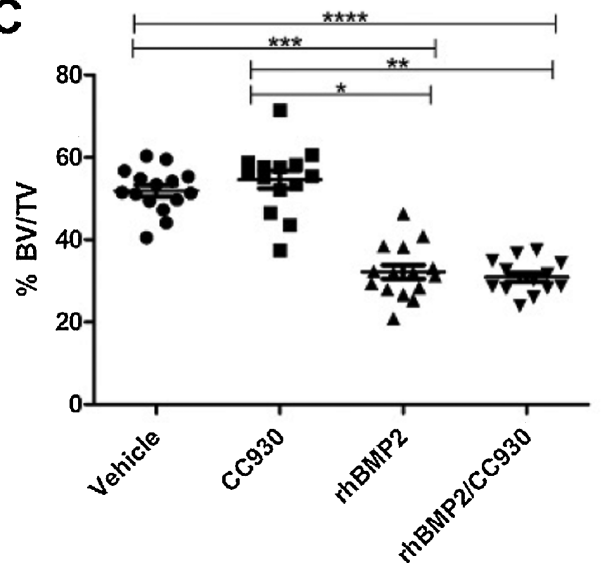

Fig. 4. rhBMP-2 enhances callus bone volume. (A) The considerable increases in callus size and bone are illustrated in microCT images of longitudinal sections through the calluses of representative specimens from each group at 3 weeks. Quantification of (B) BV and (C) BV/TV by microCT showed significant increases in BV but decreased BV/TV in rhBMP-2 treated fractures $(P<0.01)$. CC-930 did not affect callus BV or BV/TV.

in vehicle controls). All treatment groups showed reduced cartilage, indicating that no group had a delay of endochondral ossification or soft-callus breakdown.

Tissue histology revealed that CC-930 treatment reduced fibrous tissue inside the fracture gap of $N f 1^{\text {null }}$ fractures (Fig. 5C), likely underlying the improvements in union rate (Fig. 3B). In mice containing a Cre-responsive tdTomato fluorescent transgene, robust signal was seen in the fibrous tissue but not the new bone or old cortical bone (Fig. 5D). These data indicate that the fibrous cells impairing bone union are $N f 1^{\text {null }}$ cells that were successfully transduced by the Ad-Cre virus. In a separate assay to confirm CC-930 inhibition in an ex vivo model, fracture explants were cultured with CC-930 and showed a major reduction of p-cjun (JNK activity) in 2/3 samples (Supp. Fig. 1B).

\section{Discussion}

In this paper we present a series of experiments examining the effects of JNK inhibitor CC-930 on fracture healing, and later in a specific model of $N f 1^{\text {null }}$ tibial fractures. CC-930 was discovered in 2012 as an orally active anti-fibrotic JNK inhibitor (Plantevin Krenitsky et al., 2012) and has been subsequently used to treat murine models of dermal fibrosis (Reich et al., 2012) and pulmonary fibrosis (van der Velden et al., 2016). It was hypothesized that CC-930 may have multiple benefits in the context of NF1 bone repair. First, that it may enhance osteoprogenitor differentiation. Cultured $N f 1^{\text {null }}$ primary osteoblasts have been reported to show deficient matrix mineralization, which was successfully rescued by JNK inhibitor SP600125 (Sullivan et al., 2011). In addition, it was hypothesized that the pathological fibrosis associated with NF1 pseudarthrosis may be ameliorated by the anti-fibrotic properties of CC-930 and that this would improve union rates. Lastly, it was speculated that CC-930 may have additional benefits in the context of NF1 where RAS-JNK signalling is upregulated (Sullivan et al., 2011).

The data from these studies indicate that, contrary to our initial hypothesis, CC-930 does not have a notable effect on bone anabolism in healing fractures. CC-930 was found to promote matrix mineralization in cultured primary mouse embryonic fibroblasts (Supp. Fig. 1A) consistent with prior cell culture studies (Sullivan et al., 2011). However, in wild type fractures CC-930 treatment regimens did not significantly enhance callus bone volume (Fig. 1C). Although a significant difference in union was seen with D11-D14 CC-930 dosing at 2 weeks (Fig. 1B), translating a similar precise timing to a clinical context would be challenging. In the $N f 1^{\text {null }}$ fracture model, again no significant difference was seen versus vehicle controls in callus bone volume (Fig. 4B). Taken together, this would indicate that the pro-osteogenic effects of JNK inhibitors seen in culture poorly translate to an in vivo bone healing model. These findings are not unique to JNK inhibitors, with other 
A

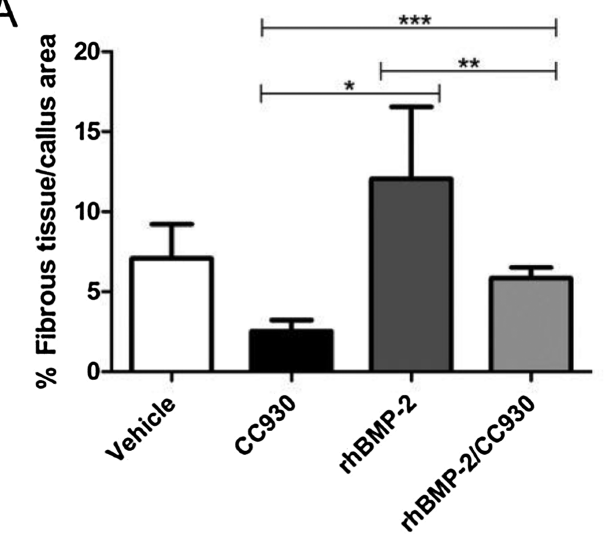

C

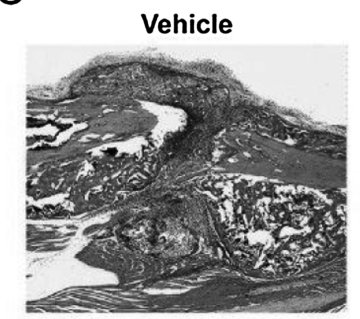

CC-930 (D11-21)

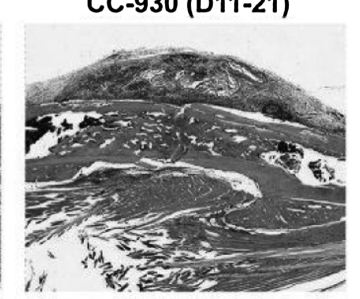

B

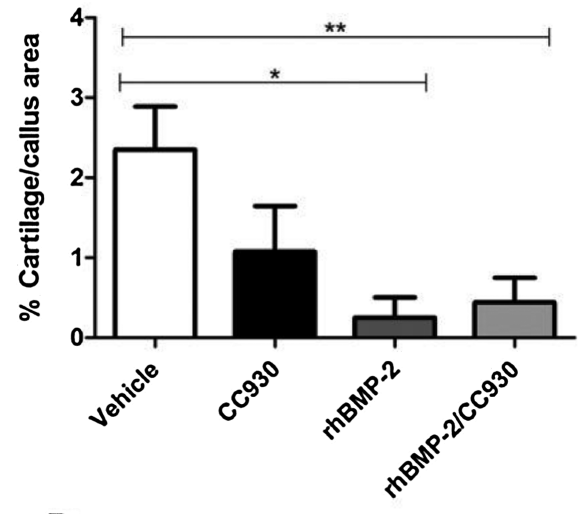

D

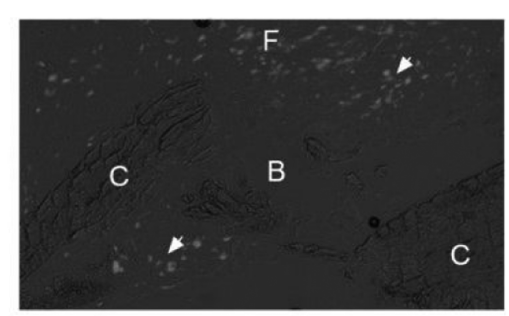

Fig. 5. Histology of Nf1-null tibial fractures and quantification of callus fibrous tissue and cartilage following 3 wk healing. Sections from multiple specimens were quantitatively analyzed for (A) fibrous and (B) cartilaginous tissue at 3 weeks. Treatment with rhBMP- 2 showed increased fibrous tissue compared to CC-930 ( ${ }^{*} P=0.02$ ) and the combination treatment $\left({ }^{* *} P<0.05\right)$. The increased fibrous tissue with rhBMP-2 was rescued by the combination treatment $\left({ }^{* * *} P<0.01\right)$. In contrast, cartilaginous tissue was significantly reduced by the rhBMP-2 and the combination therapy ( ${ }^{*} P=0.04,{ }^{* *} P<0.01$ ). (C) The reduction in fibrous tissue around the fracture site with CC930 treatment is illustrated in histological images. (D) Fluorescent imaging of tdTomato+ cells transduced with Ad-Cre virus shows abundant expression in the fibrous/cellular tissue around the fracture site [F] but not in the new bony callus [B] or old cortical bone [C].

signalling inhibitors such as TGF $\beta$ inhibitors showing a proosteogenic effect in culture that failed to be recapitulated in small animal models (Schindeler et al., 2010).

The second hypothesis, that CC-930 would reduce fibrosis at the fracture site, was supported by the study data. In the $N f 1^{\text {null }}$ fracture model, this was reflected by a decrease in fibrous tissue (Fig. 5A,C) that was associated with increased union rates (Fig. 3B). Notably, the fact that increased union was achieved despite no alteration in callus bone volume supports the concept of pathological $N f 1^{\text {null }}$ fracture tissue impeding bony bridging. Indeed, many of the unbridged, untreated $N f 1^{\text {null }}$ fractures showed accumulation of a fibrous mass in the fracture gap that was not seen in the CC-930 treated specimens (Fig. 5C). It can be contended that an increased union rate would be associated with increased mean biomechanical strength, however as a caveat this was not empirically determined by bend testing.

The use of rhBMP-2 in the treatment of NF1 pseudarthrosis has been reported on in both preclinical models (de la Croix Ndong et al., 2015; El-Hoss et al., 2014a) and in clinical case series (Birke et al., 2010; Richards et al., 2010). While the rhBMP-2 intervention has had shown beneficial results in both instances, rhBMP-2 alone does not appear to yield certainty for bone union in NF1 pseudarthrosis. Notably, in the $N f 1^{\text {null }}$ fracture model, treatment with rhBMP-2 and MEK inhibitor PD0325901 led to increased callus bone formation but also increased fibrosis (Sullivan et al., 2011). Neither intervention reduced the accumulation of fibrotic tissue, making the concept of an anti-fibrotic approach particularly appealing. The combination of CC-930 with BMP-2 reduced fibrosis, but did not affect callus bone volume or bridging. While this could lead to a prima facie argument for fibrosis being unimportant for NF1 pseudarthrosis, fibrotic tissue is routinely excised at surgery (Richards et al., 2010) and BMP-2 is not universally effective as a treatment strategy, unlike in this murine model (Birke et al., 2010; Richards et al., 2010). Thus it is speculated that a reduction of fibrosis in conjunction with BMP-2 may have benefits in terms of union rate that are not reflected in this murine model.

In wild type closed fractures, the effects of CC-930 on fibrosis were not detectable, however this was not unexpected due to the robust healing seen at the D21 time point in a model that features predictable bone union. It is possible that CC-930 may show benefits in alternative orthopedic models featuring fibrosis or delayed healing, such as open fracture, osteotomy, or gap healing models, or fractures featuring electrocautery.

The underlying mechanism of CC-930 on the prevention of rhBMP-2 associated fibrosis in NF1 deficient fracture healing was not specifically addressed by this study. However, JNK signalling shows a role in canonical TGF $\beta 1$ signalling in fibroblasts and has also been shown to regulate a JNK-TGFb-VEGF axis in osteoblasts (Kanno et al., 2005). Hyperactive TGF $\beta$-1 signalling has been found to play an important role in potentiating osseous defects in another pre-clinical model of NF1 defective bone healing (Rhodes et al., 2013). In this model, Nf1-deficient osteoblasts, a key source of TGF$\beta 1$ in bone showed both elevated secretion of and hyperresponsiveness to TGF- $\beta 1$. While the exact role of TGF- $\beta$ in NF1 tibial pseudarthrosis is clearly complex, we speculate that changes to other TGF- $\beta 1$-mediated effector pathways will be worthy of further investigation.

Finally, JNK inhibition represents a new pathway-specific approach for the treatment of NF1/CPT. To date, a number of studies have investigated agents that affect RAS prenylation such as Lovastatin for bone healing (Kolanczyk et al., 2008; Wang et al., 2010, 2011). Notably, $\operatorname{Tg} f \beta$ mRNA expression was significantly decreased by Lovastatin treatment in osteoblast-specific Nf1 knockout fractures (Wang et al., 2011). As discussed, MEK inhibition was insufficient alone to rescue $N f 1^{\text {null }}$ fracture repair 
(Sullivan et al., 2011), and even in combination with rhBMP-2 did not have any effects on fibrosis. Conversely, JNK antagonism with CC-930 reduced fibrosis without improvement of bone anabolism. Thus it is possible that the role of different Ras-dependent signalling pathways are both cell type and temporally contextual in bone healing. This would make development and translation of pathway-based approaches challenging, even if the concept of initial inhibition of MEK-MAPK followed by suppression of JNK signalling scientifically appealing.

\section{Conclusions}

These data support a key role for Ras-JNK signalling in the development of NF1 pseudarthrosis via the accrual of pathological fibrotic tissue. Suppression of JNK signalling following soft callus formation led to increased union and decreased rhBMP-2 induced fibrosis in an $N f 1^{\text {null }}$ fracture model. Moreover, in combination with rhBMP-2 to rescue insufficient bone anabolism, CC-930/rhBMP-2 led to complete union of all $N f 1^{\text {null }}$ fractures within 3 weeks.

\section{Conflict of interests}

The authors have no conflict of interests to disclose.

\section{Acknowledgements}

This work was supported by funding from the National Health and Medical Research Council of Australia (GNT1003478) and the Children's Tumour Foundation (DDI Award 2013B-05-004). Funding and in-kind support (CC930 drug and analysis of CC930 in murine serum samples) was provided by Celgene Corp. ND received funding support from the Bone Health Foundation (Australia).

\section{Appendix A. Supplementary data}

Supplementary data associated with this article can be found, in the online version, at https://doi.org/10.1016/j.jab.2018.01.006.

\section{References}

Birke, O., Schindeler, A., Ramachandran, M., Cowell, C.T., Munns, C.F., Bellemore, M., Little, D.G., 2010. Preliminary experience with the combined use of recombinant bone morphogenetic protein and bisphosphonates in the treatment of congenital pseudarthrosis of the tibia. J. Child. Orthop. 4 (6), 507-517.

Daginakatte, G.C., Gianino, S.M., Zhao, N.W., Parsadanian, A.S., Gutmann, D.H., 2008. Increased c-Jun-NH2-kinase signalling in neurofibromatosis-1 heterozygous microglia drives microglia activation and promotes optic glioma proliferation. Cancer Res. 68 (24), 10358-10366.

Davis, R.J., 2000. Signal transduction by the JNK group of MAP kinases. Cell 103 (2), 239-252.

de la Croix Ndong, J., Stevens, D.M., Vignaux, G., Uppuganti, S., Perrien, D.S., Yang, X., et al., 2015. Combined MEK inhibition and BMP2 treatment promotes osteoblast differentiation and bone healing in Nf1Osx -/- mice. J. Bone Miner. Res. 30 (1), 55-63.

El Khassawna, T., Toben, D., Kolanczyk, M., Schmidt-Bleek, K., Koennecke, I., Schell, H., et al., 2012. Deterioration of fracture healing in the mouse model of NF1 long bone dysplasia. Bone 51 (4), 651-660

El-Hoss, J., Sullivan, K., Cheng, T., Yu, N.Y.C., Bobyn, J.D., Peacock, L., et al., 2012. A murine model of neurofibromatosis type 1 tibial pseudarthrosis featuring proliferative fibrous tissue and osteoclast-like cells. J. Bone Miner. Res. 27 (1), 68-78.

El-Hoss, J., Cheng, T., Carpenter, E.C., Sullivan, K., Deo, N., Mikulec, K., et al., 2014a. A combination of rhBMP-2 (Recombinant Human Bone Morphogenetic Protein2) and MEK (MAP Kinase/ERK Kinase) inhibitor PD0325901 increases bone formation in a murine model of neurofibromatosis type I Pseudarthrosis. J. Bone Joint Surg. 96 (14), e117.

El-Hoss, J., Kolind, M., Jackson, M.T., Deo, N., Mikulec, K., McDonald, M.M., et al., 2014b. Modulation of endochondral ossification by MEK inhibitors PD0325901 and AZD6244 (Selumetinib). Bone 59, 151-161.

Elefteriou, F., Benson, M.D., Sowa, H., Starbuck, M., Liu, X., Ron, D., et al., 2006. ATF4 mediation of NF1 functions in osteoblast reveals a nutritional basis for congenital skeletal dysplasia. Cell Metab. 4 (6), 441-451.
Evans, D.G., Howard, E., Giblin, C., Clancy, T., Spencer, H., Huson, S.M., Lalloo, F., 2010 Birth incidence and prevalence of tumour-prone syndromes: estimates from a UK family genetic register service. Am. J. Med. Genet. A 152A (2), 327-332.

Han, Z., Boyle, D.L., Chang, L., Bennett, B., Karin, M., Yang, L., et al., 2001. c-Jun Nterminal kinase is required for metalloproteinase expression and joint destruction in inflammatory arthritis. J. Clin. Invest. 108 (1), 73-81.

Hefti, F., Bollini, G., Dungl, P., Fixsen, J., Grill, F., Ippolito, E., et al., 2000. Congenita pseudarthrosis of the tibia: history, etiology, classification, and epidemiologic data. J. Pediatr. Orthop. B 9 (1), 11-15.

Johnson, G.L., Lapadat, R., 2002. Mitogen-activated protein kinase pathways mediated by ERK, JNK, and p38 protein kinases. Science 298 (5600), 1911-1912.

Kanno, Y., Ishisaki, A., Yoshida, M., Tokuda, H., Numata, O., Kozawa, O., 2005. SAPK JNK plays a role in transforming growth factor-beta-induced VEGF synthesis in osteoblasts. Hormone and metabolic research. Hormones et Metab. 37 (3), 140 145.

Kolanczyk, M., Kuhnisch, J., Kossler, N., Osswald, M., Stumpp, S., Thurisch, B., et al., 2008. Modelling neurofibromatosis type 1 tibial dysplasia and its treatment with lovastatin. BMC Med. 6, 21

Madisen, L., Zwingman, T.A., Sunkin, S.M., Oh, S.W., Zariwala, H.A., Gu, H., et al., 2010 A robust and high-throughput Cre reporting and characterization system for the whole mouse brain. Nat. Neurosci. 13 (1), 133-140.

Plantevin Krenitsky, V., Nadolny, L., Delgado, M., Ayala, L., Clareen, S.S., Hilgraf, R., et al., 2012. Discovery of CC-930, an orally active anti-fibrotic JNK inhibitor. Bioorg. Med. Chem. Lett. 22 (3), 1433-1438.

Rafii, R., Juarez, M.M., Albertson, T.E., Chan, A.L., 2013. A review of current and nove therapies for idiopathic pulmonary fibrosis. J. Thoracic Dis. 5 (1), 48-73.

Reich, N., Tomcik, M., Zerr, P., Lang, V., Dees, C., Avouac, J., et al., 2012. N-terminal kinase as a potential molecular target for prevention and treatment of derma fibrosis. Ann. Rheum. Dis. 71 (June (5)), 737-745.

Rhodes, S.D., Wu, X., He, Y., Chen, S., Yang, H., Staser, K.W., et al., 2013. Hyperactive transforming growth factor-beta1 signalling potentiates skeletal defects in a neurofibromatosis type 1 mouse model. J. Bone Miner. Res. 28 (12), 2476-2489.

Richards, B.S., Oetgen, M.E., Johnston, C.E., 2010. The use of rhBMP-2 for the treatment of congenital pseudarthrosis of the tibia: a case series. J. Bone Joint Surg. 92 (1), 177-185.

Schindeler, A., Little, D.G., 2008. Recent insights into bone development, homeostasis, and repair in type 1 neurofibromatosis (NF1). Bone 42 (4), 616 622.

Schindeler, A., Morse, A., Harry, L., Godfrey, C., Mikulec, K., McDonald, M., et al. 2008a. Models of tibial fracture healing in normal and Nf1-deficient mice. J. Orthop. Res. 26 (8), 1053-1060.

Schindeler, A., Ramachandran, M., Godfrey, C., Morse, A., McDonald, M., Mikulec, K. Little, D.G., 2008b. Modeling bone morphogenetic protein and bisphosphonate combination therapy in wild-type and Nf1 haploinsufficient mice. J. Orthop. Res. 26 (1), 65-74.

Schindeler, A., Morse, A., Peacock, L., Mikulec, K., Yu, N.Y., Liu, R., et al., 2010. Rapid cell culture and pre-clinical screening of a transforming growth factor-beta (TGF-beta) inhibitor for orthopaedics. BMC Musculoskeletal Disord. 11, 105.

Sharma, R., Wu, X., Rhodes, S.D., Chen, S., He, Y., Yuan, J., et al., 2013. Hyperactive Ras/ MAPK signalling is critical for tibial nonunion fracture in neurofibromindeficient mice. Hum. Mol. Genet. 22 (23), 4818-4828.

Stevenson, D.A., Zhou, H., Ashrafi, S., Messiaen, L.M., Carey, J.C., D’Astous, J.L., et al., 2006. Double inactivation of NF1 in tibial pseudarthrosis. Am. J. Hum. Genet. 79 (1), 143-148.

Stevenson, D.A., Moyer-Mileur, L.J., Murray, M., Slater, H., Sheng, X., Carey, J.C., et al., 2007. Bone mineral density in children and adolescents with neurofibromatosis type 1. J. Pediatr. 150 (1), 83-88.

Stevenson, D.A., Little, D., Armstrong, L., Crawford, A.H., Eastwood, D., Friedman, J.M., et al., 2013. Approaches to treating NF1 tibial pseudarthrosis: consensus from the children's tumour foundation NF1 bone abnormalities consortium. J. Pediatr. Orthop. 33 (3), 269-275.

Sullivan, K., El-Hoss, J., Little, D.G., Schindeler, A., 2011. JNK inhibitors increase osteogenesis in Nf1-deficient cells. Bone 49 (6), 1311-1316.

Tournier, C., Hess, P., Yang, D.D., Xu, J., Turner, T.K., Nimnual, A., et al., 2000. Requirement of JNK for stress-induced activation of the cytochrome c-mediated death pathway. Science 288 (5467), 870-874.

van der Velden, J.L., Ye, Y., Nolin, J.D., Hoffman, S.M., Chapman, D.G., Lahue, K.G., et al., 2016. JNK inhibition reduces lung remodelling and pulmonary fibrotic systemic markers. Clin. Transl. Med. 5 (1), 36.

Wang, W., Nyman, J.S., Moss, H.E., Gutierrez, G., Mundy, G.R., Yang, X., Elefteriou, F., 2010. Local low-dose lovastatin delivery improves the bone-healing defect caused by Nf1 loss of function in osteoblasts. J. Bone Miner. Res. 25 (7), 16581667.

Wang, W., Nyman, J.S., Ono, K., Stevenson, D.A., Yang, X., Elefteriou, F., 2011. Mice lacking Nf1 in osteochondroprogenitor cells display skeletal dysplasia similar to patients with neurofibromatosis type I. Hum. Mol. Genet. 20 (20), 3910-3924

Zha, Y., Shah, R., Locke, F., Wong, A., Gajewski, T.F., 2008. Use of cre-adenovirus and CAR transgenic mice for efficient deletion of genes in post-thymic $T$ cells J. Immunol. Methods 331 (1-2), 94-102.

Zhu, Y., Romero, M.I., Ghosh, P., Ye, Z., Charnay, P., Rushing, E.J., et al., 2001. Ablation of NF1 function in neurons induces abnormal development of cerebral cortex and reactive gliosis in the brain. Genes Dev. 15 (7), 859-876. 